\title{
Relay Auction Algorithms for Downlink Bandwidth Allocation in IEEE 802.16-Based OFDM/TDMA Wireless Mesh Networks
}

\author{
Zhen Kong ${ }^{\ddagger}$ and Yu-Kwong Kwok ${ }^{\dagger \ddagger}$ \\ †The University of Hong Kong, Hong Kong \\ ${ }^{\ddagger}$ Colorado State University, Fort Collins, CO 80526, USA
}

\begin{abstract}
In this paper, we study the problem of downlink bandwidth allocation in a non-cooperative IEEE 802.16 OFDM/TDMA based wireless mesh network, and propose an auction based framework in which the gateway, equipped with the precious high speed Internet connection, serves as the auctioneer while the first-level mesh routers (MRs) (i.e., those with direct wireless connections to the gateway) act as bidders competing resources among each other. We then present two novel relay auction (RA) approaches to allocate time-slots among MRs and analyze the bidding strategy using the solution concept of Nash equilibrium. Through simulations, we find that the proposed RA algorithms can achieve competitive performance in terms of resource allocation efficiency compared with Vickrey-ClarkeGroves (VCG) approaches, while having the capability to strike a proper balance between efficiency and fairness by adjusting the payment function. Specifically, with a smaller payment parameter value, the RA algorithms can also achieve much better connection blocking probability performance than VCG algorithms.
\end{abstract}

Index Terms-Wireless mesh networks, downlink bandwidth allocation, auction algorithms, Nash equilibrium, VickreyClarke-Groves auction, service dependability.

\section{INTRODUCTION}

As a means to provide universal Internet access services at affordable monetary and infrastructure costs in rural areas, the research and deployment of IEEE 802.16-based WMNs have gained enormous popularity recently [1], [11]. A typical WMN consists of mesh routers (MRs) that not only provide wireless access for mesh clients (MCs) but also form the backbone of the network. To provide broadband out-bound access, gateways are usually installed between the mesh backbone and the Internet. Consequently, as shown in Fig. 1, to provide broadband Internet services to a remote area, the MRs close to the gateway can work as relay nodes transmitting traffic between the Internet and the MRs far away from the gateway.

One of the key enabling features behind such a hierarchical WMN is that a MR should not only serve the MCs in its own local cell, but also relay traffic for outer level MRs. Unfortunately, this kind of cooperation among MRs is not always practical in reality, especially when these MRs are owned by different profit-maximizing entities so that they have to compete with each other for radio resource. In such a non-cooperative environment, a MR will rationally exhibit selfish behaviors driven by self-interests in that the MR may

Corresponding Author: Y.-K. Kwok (Email: ykwok@hku.hk)

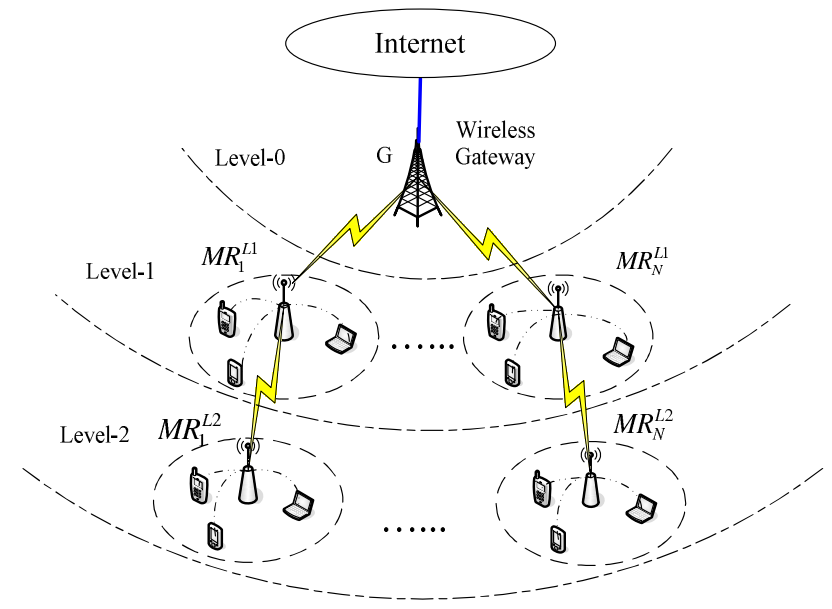

Fig. 1: Structure of a hierarchical wireless mesh network.

report a bogus channel information or valuation so as to improve its own benefit [7], or refuse to relay other MRs' traffic because relaying traffic will inevitably consume its own resource and consequently degrade the performance of its local cell. Though these non-cooperative behaviors could improve the performance for the selfish MR itself, they may lead to inefficient or unfair resource utilization for the whole system.

Several recent works [5], [12] have addressed the problem of resource allocation in WMNs, but they are either based on a cooperative situation or the assumption that the relay node itself does not have local connections to serve, which are different from our model where a relay MR should serve both local and relay connections in a non-cooperative environment.

The resource allocation problem in a competitive environment can be effectively addressed by means of auction theory [9], which is widely known to be efficient in allocating resources in a non-cooperative situation, and is the focus of our study. Specifically, in this hierarchical mesh infrastructure, we assume using an IEEE 802.16 WirelessMAN-OFDM air interface, where the physical layer is based on orthogonal frequency-division multiplexing (OFDM), and the media access control (MAC) scheme is based on time-division multiple access (TDMA). With OFDM/TDMA, all subchannels are allocated to one connection at a time. Thus, in our model, the auction goods are time-slots, the auctioneer is the gateway who has radio resource, i.e., time-slots, to allocate, and the bidders 
are MRs who need to request time-slots from the gateway and use them for local or relay traffic transmissions.

To implement an auction approach in WMNs, two challenging problems must be tackled. First, a succinct and expressive bidding language is necessary. Here, we define a bidder's valuation function by quantifying its valuation on the allocated resource under the current state, and then fully characterize it simply by a single scalar parameter. Thus, each bidder can submit the scalar as its bid, thereby leading to an efficient and practical bidding process. Second, how should we define "payment"? In some auction-based algorithms [5], [14], payments are expressed in terms of money or some kinds of virtual parameters, and thus, suffer from a lack of practical meanings and significance. Here we consider associating payment with the time-slots used by an MR to relay other MR's packets. Specifically, to win time-slots allocated by the gateway, a bidder must "pay" some wireless resources, i.e., time-slots, and use them to forward an outer level MR's traffic.

In our previous work [8], we have proposed two kinds of auction methods based on the well known Vickrey-ClarkeGroves (VCG) model [9]. Unfortunately, there is one severe drawback in VCG-based algorithms in that they do not consider fairness performance [2]. This is clearly undesirable in a wireless resource allocation environment where the gateway should provide some level of QoS guarantees in terms of fairness. To overcome the drawback of the VCG algorithms, in this paper, we propose a novel relay auction (RA) framework based on a concave utility function, where the bid submitted by a MR is a scalar associated with its reported valuation, while the payment is increasing with this bidding scalar value. Specifically, depending on the type of payment function, i.e., linear or logarithmic payment function, we design two RA algorithms, called RALiP and RALoP. We first prove the existence and uniqueness of Nash equilibrium (NE) [13]. We then compare the performance of these auction algorithms through simulations, and find that the proposed RA algorithms can achieve aggregate throughput performance comparable to VCG approach, while they can adjust the resource ratio allocated to different level of MRs, so as to strike a proper balance between efficiency and fairness. From a service dependability perspective, the performance of our RA algorithms is also better than VCG in terms of connection blocking probability.

The rest of this paper is organized as follows. We present the system model and auction model in Section II. In Section III, our novel relay auction approaches are proposed. Section IV gives the simulation results. We conclude in Section V.

\section{MODEL}

\section{A. System Model}

In this paper, we consider an IEEE 802.16 OFDM/TDMAbased WMN with three hierarchical levels as shown in Fig. 1 and focus on downlink resource allocation. Such a hierarchical network structure is highly practical and can be used to model a $\mathrm{WMN}$ in a rural area, where at Level-0, there is a wireless gateway that has direct connection to the Internet. There are $N$ Level-1 MRs, denoted by $M R_{i}^{L_{1}}, i \in \mathbb{N}=\{1,2, \ldots, N\}$, surrounding the gateway and providing wireless access for their clients. Each MR and its corresponding clients form a cell. Outside Level-1, to cover a wide geographical area (a necessary condition in a rural area), there are $N$ other MRs, denoted by $M R_{i}^{L_{2}}$, which are located far from the central gateway. As a result, each $M R_{i}^{L_{2}}$ does not have direct wireless connections with the gateway, and it can only get broadband Internet services through the relaying services provided by $M R_{i}^{L_{1}}$. We also assume that $M R_{i}^{L_{1}}$ in Level-1 just relays traffics to $M R_{i}^{L_{2}}$ in Level-2. Every Level-1 $M R_{i}^{L_{1}}$ competes with each other for the bandwidth resource provided by the gateway.

We assume all cells operate under IEEE 802.16 OFDM/TDMA-TDD mode, and each adjacent cell uses a different frequency band. The MAC frame is composed of downlink and uplink subframes. Each downlink subframe is composed of $T_{d}$ time-slots, each of which is used for transmission of packets corresponding to one connection. For uplink and downlink transmission using OFDM, each MR uses $M$ subchannels with total bandwidth of $B \mathrm{MHz}$. There are $R_{i}^{G L_{1}}$ packets per time-slot transmitted from the gateway to $M R_{i}^{L_{1}}$. We use $P E R_{i}^{L_{1}}$ and $P E R_{i}^{L_{2}}$ to denote the average packet error rates in the downlink of $M R_{i}^{L_{1}}$,s and $M R_{i}^{L_{2}}$, s local cell, respectively. These PERs depend on the PHY layer packet transmission error rate and MAC layer packet dropping rate. Then we can get the average number of packets that can be transmitted successfully per time-slot from $M R_{i}^{L_{1}}$ and $M R_{i}^{L_{2}}$ as $a_{i}^{L_{1}}$ and $a_{i}^{L_{2}}$, respectively, i.e.,

$$
\begin{gathered}
a_{i}^{L_{1}}=R_{i}^{G L_{1}} \cdot\left(1-P E R_{i}^{L_{1}}\right) \\
a_{i}^{L_{2}}=a_{i}^{L_{1}} \cdot\left(1-P E R_{i}^{L_{2}}\right)
\end{gathered}
$$

We also assume that there are $N_{i}^{L_{1}} \mathrm{MCs}$ served by $M R_{i}^{L_{1}}$, and $N_{i}^{L_{2}}$ MCs served by $M R_{i}^{L_{2}}$. Thus, when $T_{i}^{L_{1}}$ time-slots are allocated to $M R_{i}^{L_{1}}$ for its local usage and $T_{i}^{L_{2}}$ time-slots are used to relay Level-2 MR's traffic, the average received packets per frame by a Level-1 MC and a Level-2 MC are then given by:

$$
\begin{aligned}
& \bar{Q}_{i}^{L_{1}}\left(T_{i}^{L_{1}}\right)=\frac{a_{i}^{L_{1}}}{N_{i}^{L_{1}}} \cdot T_{i}^{L_{1}} \\
& \bar{Q}_{i}^{L_{2}}\left(T_{i}^{L_{2}}\right)=\frac{a_{i}^{L_{2}}}{N_{i}^{L_{2}}} \cdot T_{i}^{L_{2}}
\end{aligned}
$$

\section{B. Auction Framework}

In our proposed auction framework, each $M R_{i}^{L_{1}}$ needs to compete for time-slots through auction, and the valuation function can be fully characterized by a scalar valuation parameter. Specifically, every $M R_{i}^{L_{1}}$ calculates the valuation parameter $a_{i}^{L_{1}}$ and then submits a bid $b_{i}=\mu_{i}\left(a_{i}^{L_{1}}\right)$ to the gateway according to a rationally selfish strategy. For $M R_{i}^{L_{2}}$, because $a_{i}^{L_{2}}$ depends on $P E R_{i}^{L_{2}}$ and $M R_{i}^{L_{1}}$ 's reported valuation parameter, we assume that every $M R_{i}^{L_{2}}$ can send $P E R_{i}^{L_{2}}$ to the gateway via a secure out-of-band control channel through $M R_{i}^{L_{1}}$. After receiving the announced bidding profile $B=\left\{b_{1}, b_{2}, \ldots, b_{N}\right\}$ from every $M R_{i}^{L_{1}}$, and all level-2 $P E R_{i}^{L_{2}}$, the gateway will know each MR's reported valuation, and then calculate the allocation $T=$ 
$T(B)=\left\{T_{1}, T_{2}, \ldots, T_{i}, \ldots, T_{N}\right\}$, which represents the number of time-slots allocated to each $M R_{i}^{L_{1}}$, as well as the payment $P=P(B)=\left\{T_{1}^{L_{2}}, T_{2}^{L_{2}}, \ldots, T_{i}^{L_{2}}, \ldots, T_{N}^{L_{2}}\right\}$, which represents the number of time-slots that should be used by $M R_{i}^{L_{1}}$ to relay $M R_{i}^{L_{2}}$ 's traffic. Subsequently, the allocation and payment results are transmitted to each $M R_{i}^{L_{1}}$. Upon receiving them, each $M R_{i}^{L_{1}}$ gets to know $T_{i}^{L_{1}}=T_{i}-T_{i}^{L_{2}}$ and payment $T_{i}^{L_{2}}$.

In a typical auction, the utility of a bidder is the value received by this bidder minus the payment assigned by the auctioneer. However, in our model, to provide practical meanings for payment, we associate the payment with the practical radio resource, i.e., time-slots. Thus, when $M R_{i}^{L_{1}}$ receives $T_{i}$ time-slots and accept $T_{i}^{L_{2}}$ time-slots as payment, its ultimate utility is its true valuation when getting $T_{i}^{L_{1}}=T_{i}-T_{i}^{L_{2}}$, i.e.,

$$
U_{i}^{L_{1}}\left(T_{i}, T_{i}^{L_{2}}\right)=V_{i}^{L_{1}}\left(T_{i}-T_{i}^{L_{2}}\right)=V_{i}^{L_{1}}\left(T_{i}^{L_{1}}\right)
$$

For Level-2 $M R_{i}^{L_{2}}$, its utility is just its valuation with $T_{i}^{L_{2}}$ time-slots, i.e.,

$$
U_{i}^{L_{2}}\left(T_{i}^{L_{2}}\right)=V_{i}^{L_{2}}\left(T_{i}^{L_{2}}\right)
$$

Specifically, we consider the following logarithmic function to express a level-1 MC's valuation on its average received packets per frame, i.e.,

$$
v_{i}^{L_{1}}\left(a_{i}^{L_{1}}, T_{i}^{L_{1}}\right)=\log \left(\bar{Q}_{i}^{L_{1}}\left(T_{i}^{L_{1}}\right)+1\right)=\log \left(\frac{a_{i}^{L_{1}}}{N_{i}} \cdot T_{i}^{L_{1}}+1\right)
$$

Thus, when there are $N_{i}^{L_{1}}$ MCs served by $M R_{i}^{L_{1}}$, its valuation on $T_{i}^{L_{1}}$ time-slots can be defined as: $V_{i}^{L_{1}}\left(a_{i}^{L_{1}}, T_{i}^{L_{1}}\right)=N_{i}^{L_{1}} \cdot v_{i}^{L_{1}}\left(a_{i}^{1}, T_{i}^{L_{1}}\right)=N_{i}^{L_{1}} \cdot \log \left(\frac{a_{i}^{L_{1}}}{N_{i}^{L_{1}}} \cdot T_{i}^{L_{1}}+1\right)$

Similarly, $M R_{i}^{L_{2}}$ 's valuation on $T_{i}^{L_{2}}$ time-slots is:

$$
V_{i}^{L_{2}}\left(a_{i}^{L_{2}}, T_{i}^{L_{2}}\right)=N_{i}^{L_{2}} \cdot \log \left(\frac{a_{i}^{L_{2}}}{N_{i}^{L_{2}}} \cdot T_{i}^{L_{2}}+1\right)
$$

Thus, according to (5) and (6), their utilities are:

$$
\begin{gathered}
U_{i}^{L_{1}}\left(T_{i}, T_{i}^{L_{2}}\right)=V_{i}^{L_{1}}\left(a_{i}^{L_{1}}, T_{i}-T_{i}^{L_{2}}\right) \\
U_{i}^{L_{2}}\left(T_{i}^{L_{2}}\right)=V_{i}^{L_{2}}\left(a_{i}^{L_{2}}, T_{i}^{L_{2}}\right)
\end{gathered}
$$

Obviously, $U_{i}^{L_{1}}$ is concave on both valuation parameter $a_{i}^{L_{1}}$ and allocated resource $T_{i}^{L_{1}}$, while $U_{i}^{L_{2}}$ is also concave on both $a_{i}^{L_{2}}$ and $T_{i}^{L_{2}}$.

\section{RELAy AuCTION}

Generally, the more resource a bidder requests, the higher payment it should pay to the auctioneer. Thus, we define the payment as a function increasing with the bidding scalar $b_{i}$, and propose a relay auction (RA) algorithm, which is formalized as follows.

\section{A. Auctioning Mechanism}

1) Bidding rule: $M R_{i}^{L_{1}}$ submits a scalar value $b_{i}$ to the gateway, and let $\mathbf{b}=\left\{b_{i}: b_{\min } \leq b_{i} \leq b_{\max }\right\}$. We also let $M R_{i}^{L_{2}}$ send its average packet error rate $P E R_{i}^{L_{2}}$ to the gateway via a secure out-of-band control channel through $M R_{i}^{L_{1}}$. Then, the gateway can know $M R_{i}^{L_{2}}$ 's valuation as $a_{i}^{L_{2}}=b_{i} \cdot\left(1-P E R_{i}^{L_{2}}\right)$.

2) Payment rule: If $M R_{i}^{L_{1}}$ gets $T_{i}$ time-slots, it must use $T_{i}^{L_{2}}=f\left(b_{i}\right) \cdot T_{i}$ time-slots for relaying $M R_{i}^{L_{2}}$ 's traffic, where $f\left(b_{i}\right)$ is increasing with $b_{i}$, and $0 \leq f\left(b_{i}\right) \leq 1$. Here, we consider two different payment functions: $f_{1}\left(b_{i}\right)=\frac{b_{i}}{A}$, and $f_{2}\left(b_{i}\right)=\frac{\log \left(b_{i}+1\right)}{A}$, where $A$ is a payment parameter.

3) Allocation rule:

$$
\begin{aligned}
T_{*} & =\left\{T_{1}^{*}, T_{2}^{*}, \cdots, T_{N}^{*}\right\}=\arg \max _{\left\{T_{i}\right\}} \sum_{i} U_{i}^{L_{1}}\left(T_{i}\right) \\
& =\arg \max _{\left\{T_{i}\right\}} \sum_{i}\left(N_{i}^{L_{1}} \cdot \log \left(\frac{b_{i}}{N_{i}^{L_{1}}} \cdot\left(T_{i}-T_{i}^{L_{2}}\right)+1\right)\right. \\
& \left.+N_{i}^{L_{2}} \cdot \log \left(\frac{b_{i} \cdot\left(1-P E R_{i}^{L_{2}}\right)}{N_{i}^{L_{2}}} \cdot T_{i}^{L_{2}}+1\right)\right)
\end{aligned}
$$

subject to:

$$
\text { (I) } \sum_{i}\left(T_{i}^{L_{1}}+T_{i}^{L_{2}}\right)=T_{d} \quad(I I) T_{i}^{L_{2}}=f\left(b_{i}\right) \cdot T_{i}
$$

\section{B. Nash Equilibrium}

Our proposed relay auction can be formulated as a strategic non-cooperative game, i.e., $\Gamma \triangleq\left[\mathbb{N},\left\{b_{i}\right\}_{i \in \mathbb{N}},\left\{U_{i}^{L_{1}}(\mathbf{B})\right\}_{i \in \mathbb{N}}\right]$, with $N$ players, where $\mathbb{N} \triangleq\{1, \cdots, N\}$ is the player set, $\left\{b_{1}, \cdots, b_{N}\right\}$ is the action profile, and $U_{i}^{L_{1}}$ is the utility

A useful solution concept of such a game is called a Nash equilibrium (NE) [13], which is a bidding profile $B^{*}$ where no MR wants to deviate unilaterally, i.e.,

$$
U_{i}^{L_{1}}\left(b_{i}^{*} ; \mathbf{b}_{-i}^{*}\right) \geq U_{i}^{L_{1}}\left(b_{i} ; \mathbf{b}_{-i}^{*}\right), \quad \forall i \in \mathbb{N}, \quad \forall b_{i} \geq 0
$$

We first establish the existence of the NE in our proposed relay auction.

Theorem 1. There exists a bidding profile $B^{*}=\left\{b_{i}^{*}, \cdots, b_{N}^{*}\right\}$ to achieve Nash equilibrium in the relay auction.

Proof: According to [3] and [4], a Nash equilibrium $B^{*}$ exists in the game if the following two conditions are satisfied for all $i=1, \cdots, N$ :

1) the bidding strategy $b_{i}$ is a nonempty, convex and compact subset of some Euclidean space $\Re^{N}$; and

2) $U_{i}^{L_{1}}(\mathbf{B})$ is continuous in $\mathbf{B}$ and quasi-concave in $b_{i}$ The first condition is easily satisfied because we have $b_{i} \in$ $\left[b_{\min }, b_{\max }\right]$. For utility function $U_{i}^{L_{1}}$, because $\frac{\partial^{2} U_{i}^{L_{1}}}{b_{i}^{2}}<0$, it is strictly concave on $b_{i}$. As a concave function is also quasi-concave, the second condition is then satisfied. Thus, the existence of $\mathrm{NE}$ is proven.

Having established the existence of NE, we further investigate the uniqueness of this NE bidding profile in relay auction. 
To this end, we first construct $M R_{i}^{L_{1}}$ 's best response function (for fixed $\mathbf{b}_{-i}$ ) as:

$$
\beta_{i}\left(\mathbf{b}_{-i}\right)=\left\{b_{i} \mid b_{i}=\arg \underset{\widetilde{b}_{i} \geq 0}{\max } U_{i}^{L_{1}}\left(\widetilde{b}_{i} ; \mathbf{b}_{-i}\right)\right\}
$$

Then, we have the following theorem.

Theorem 2. There exists a unique $N E B^{*}=\left\{b_{i}^{*}, \cdots, b_{N}^{*}\right\}$ in the relay auction.

Proof: We assume that NE is not unique, and there exists at least another one $\tilde{B}=\left\{\tilde{b}_{1}, \cdots, \tilde{b}_{N}\right\}$, and $B^{*} \neq \tilde{B}$. According to the definitions of NE and the best response function, $p_{i}^{*}$ and $\tilde{p}_{i}$ must satisfy:

$$
\begin{aligned}
b_{i}^{*} & =\max _{b_{i}^{*}} U_{i}^{L_{1}}\left(b_{i}^{*}, b_{-i}^{*}\right) \\
\tilde{b}_{i} & =\max _{\tilde{b_{i}}} U_{i}^{L_{1}}\left(\tilde{b}_{i}, \tilde{b}_{-i}\right)
\end{aligned}
$$

However, because $U_{i}^{L_{1}}$ is concave and differentiable, for a fixed $b_{-i}$, there is a unique best strategy $b_{i}$ that satisfies $b_{i}=\max _{b_{i}} U_{i}^{L_{1}}\left(b_{i}, b_{-i}\right)$. These contradict the assumption that $B^{*} \neq \tilde{B}$. Thus, $B^{*}=\tilde{B}$, and there is a unique NE in this auction.
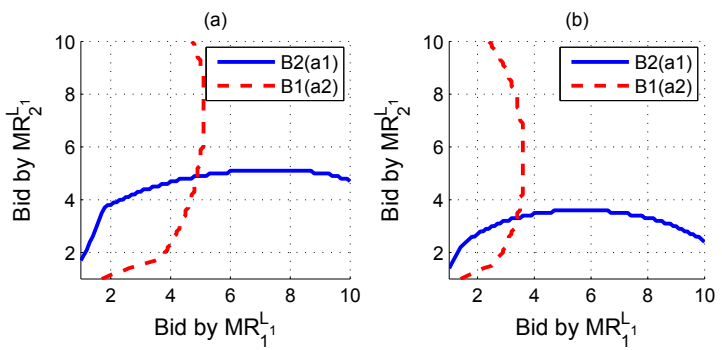

Fig. 2: Best response functions: (a) payment function is $f_{1}\left(b_{i}\right)=\frac{b_{i}}{A}$; and (2) payment function is $f_{2}\left(b_{i}\right)=\frac{\log \left(b_{i}+1\right)}{A}$.

Fig. 2 graphically depicts the existence and uniqueness of NE in case of $N=2$, where $a_{1}^{L_{2}}=a_{2}^{L_{2}}=10$, and $A=20$.

\section{Performance Results}

In this section, we provide simulations results to evaluate the performance of the auction algorithms.

\section{A. Simulation Configuration}

The simulation topology is shown in Fig. 1. Specifically, we assume each MR has 64 subcarriers with a total frequency band of $10 \mathrm{MHz}$. The frequency bands for adjacent MRs are non-coverlapping and frequency reuse is utilized. Consequently, the OFDM symbol time is $8 \mu \mathrm{s}$, in which $1.6 \mu \mathrm{s}$ is the guard interval. We further group 25 OFDM symbols into a slot, and let one DL-subframe have 10 time-slots. Thus, the DL-subframe length is $2 \mathrm{~ms}$. The wireless channel is modeled as six-path frequency-selective Rayleigh slow fading channel; each path is simulated by Clark's fading model and suffers from different Rayleigh fading with the maximum Doppler frequency of $30 \mathrm{~Hz}$. Because the coherence time $33.3 \mathrm{~ms}$ is much larger than the DL-subframe length, the channel condition can be regarded as static during the iterative bidding period to achieve NE. This is a realistic assumption in a
WMN because the MRs are immobile. We also assume that the power delay profile is exponentially decaying with $e^{-2 l}$, where $l$ is the multipath index. The AWGN power density is $-80 \mathrm{dBW} / \mathrm{Hz}$. Furthermore, the channel corresponding to different users has the same but independent statistics. We assume the average SNR is identical for all subcarriers to a particular connection, but the transmission rate in different subcarrier is different when adaptive modulation is performed independently in each subcarrier.

We simulate CVCG (a classical VCG-based auction algorithm based on a linear utility function as proposed in [8] ), QVCG (an auction approach applying the VCG allocation and payment policies to a non-linear environment as proposed in [8]), RALiP (our RA algorithm with linear payment function $f_{1}\left(b_{i}\right)=\frac{b_{i}}{A}$ ), and RALoP (our RA algorithm with logarithmic payment function $\left.f_{1}\left(b_{i}\right)=\frac{\log \left(b_{i}+1\right)}{A}\right)$. We further assume with proper scheduling and queuing policies as well as adaptive modulation used in the MR, the packet error rate in $M R_{i}^{L_{1}}$ and $M R_{i}^{L_{2}}$ are all maintained at a constant value, and $P E R_{i}^{L_{1}}=1 \%$ and $P E R_{i}^{L_{2}}=1 \%$. In this setting, $N=2$ and there are $10 \mathrm{MCs}$ in each cell.

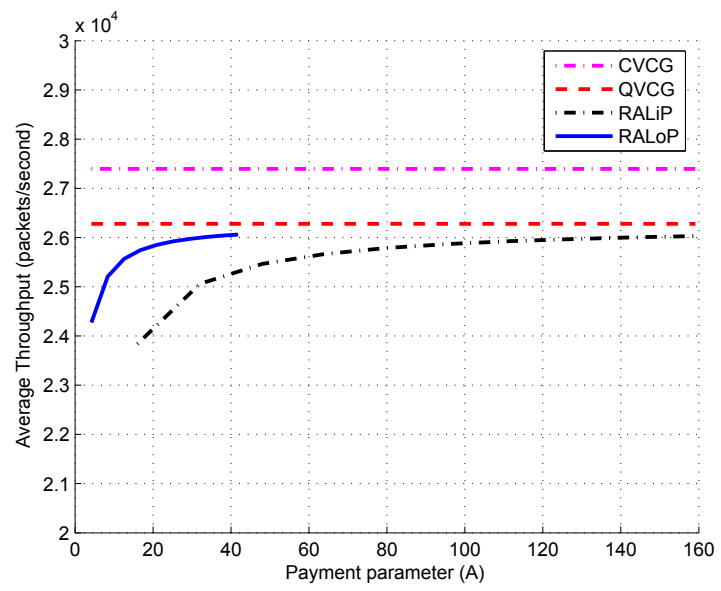

Fig. 3: System average throughput vs. payment parameter.

Fig. 3 illustrates the system average throughput performance of the different four auction algorithms considered in our study. We can see that although VCG-based approaches generate higher throughput than those of the RA algorithms, the throughput of RALiP and RALoP achieve almost $95 \%$ of that for CVCG with the increase of payment parameter. This can be explained by the fact that the time-slots used by Level-1 MR to relay Level-2 MR's packets decrease if the payment parameter becomes higher. Because Level-1 MRs are close to the gateway and generally have better transmission conditions than those of Level-2 MRs, the overall system throughput increases when more resources are used for Level-1 MRs's traffic. On the other hand, RALoP is superior to RALiP. This is because the logarithmic payment function used by RALoP makes Level-1 MR pay less given the same bids, and thus, a larger portion of resource is allocated to $M R_{i}^{L_{1}}$.

Now let us consider the fairness performance in terms of Jain fairness index [6] in Fig. 4. We can see that the Jain 
fairness indices for RALiP and RALoP are highly dependent on the payment parameter $A$. RALiP achieves its highest index value when $A=32$, while RALoP's fairness achieves its peak when $A=8$, and both are larger than that of CVCG and QVCG. Thus, the proposed RA algorithms can be used to strike a proper balance between efficiency and fairness, and achieve better fairness performance than VCG, while maintaining good efficiency performance.

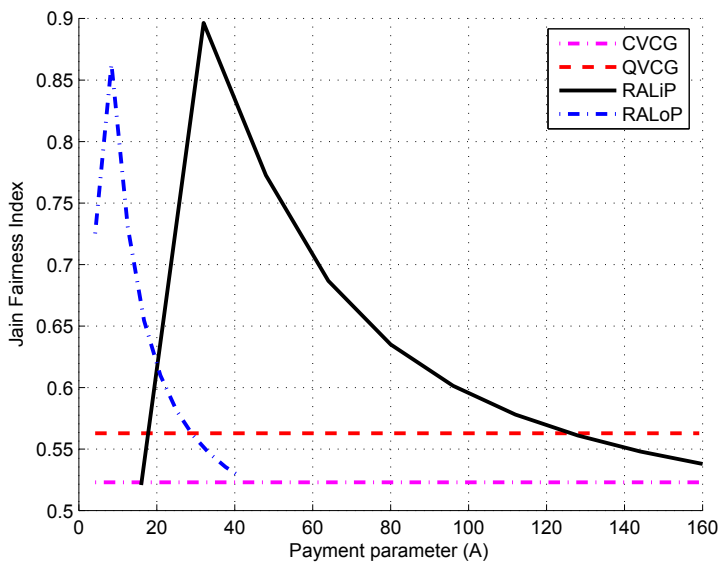

Fig. 4: Jain fairness index vs. payment parameter.

To quantify the service dependability for Level-2 MRs in the system, we assume that each local connection generates 2500 packets per second in the downlink, and calculate the Level2 MR's connection blocking probability (CBP) according to Engset formula [10]. The Level-2 MRs' average CBP performance is shown in Fig. 5. As discussed earlier, a higher payment parameter means that fewer time-slots can be used to relay Level-2 MRs'traffic. Thus, CBP values of RALiP and RALoP increase with $A$. When $A$ is relatively larger, such as $A>110$, the CBP values of RALiP and RALoP are higher than those of CVCG and QVCG. On the other hand, when $A<62$ for RALiP and $A<15$ for RALoP, the RA algorithms have better CBP performance than VCG methods, demonstrating that they have a higher service dependability.

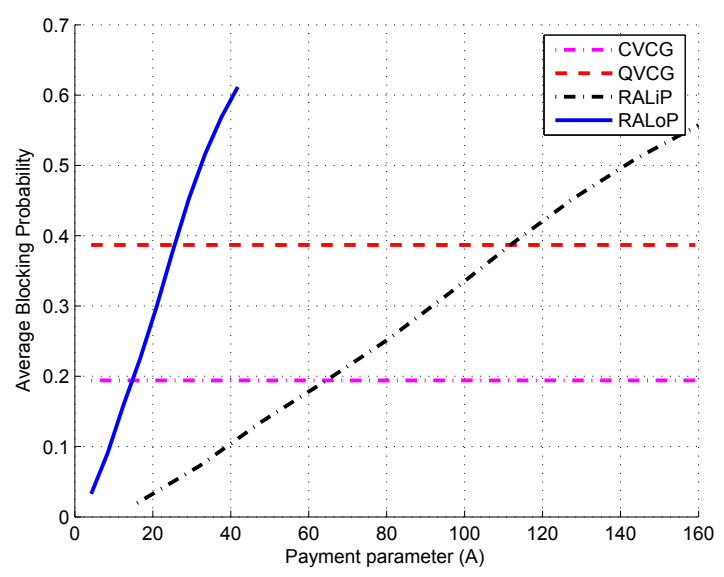

Fig. 5: Level-2 CBP vs. payment parameter.

\section{CONCLUSiOnS}

In this paper, we study the downlink bandwidth allocation problem in a non-cooperative IEEE 802.16 OFDM/TDMAbased hierarchical wireless mesh network. We design an auction framework to let rationally selfish mesh routers request time-slots actively and motivate them to forward an outer level MR's traffic. Based on this framework, we propose novel relay auction algorithms and analyze the bidding strategy using the solution concept of Nash equilibrium. After proving the existence and uniqueness of NE, we study the performance of the RA auction algorithms with our previously proposed VCG algorithms through simulations, which indicate that the proposed relay auction algorithms can achieve competitive performance in terms of resource allocation efficiency as the VCG approaches, while being able to adjust the resource ratio allocated to different levels of mesh routers, so as to strike a proper balance between efficiency and fairness. With a smaller payment parameter, the relay auction algorithms can also achieve a higher service dependability compared with the VCG algorithms.

\section{REFERENCES}

[1] I. F. Akyildiz and X. Wang, "A Survey on Wireless Mesh Networks," IEEE Communications Mag., vol. 43, no. 9, pp. S23-S30, Sept. 2005.

[2] L. M. Ausubel and P. Milgrom, "The Lovely but Lonely Vickrey Auction," In Combinatorial Auctions, P. Cramton, Y. Shoham, and R. Steinberg (eds.), Chapter 1, pp. 17-40, MIT Press, Cambridge, MA, 2006.

[3] D. Fudenberg and J. Tirole, Game Theory, MIT Press, Cambridge, MA; London, England, 1991.

[4] G. He, S. Gault, M. Debbah, and E. Altman,"Distributed Power Allocation Game for Uplink OFDM Systems," Proceedings of 6th International Symposium on Modeling and Optimization in Mobile, Ad Hoc, and Wireless Networks and Workshops (WiOPT 2008), pp. 515-521, Mar.Apr. 2008.

[5] J. Huang, Z. Han, M. Chiang, and H. V. Poor, "Auction-Based Resource Allocation for Cooperative Communications," IEEE J. Select. Areas Communications, vol. 26, no. 7, pp.1226-1237, Sept. 2008.

[6] R. Jain, D. Chiu and W. Hawe, "A Quantitative Measure of Fairness and Discrimination for Resource allocation in Shared Computer System," DEC Technical Report 301, 1984.

[7] Z. Kong, Y.-K. Kwok, and J. Wang, "On the Impact of Selfish Behaviors in Wireless Packet Scheduling," Proceedings of the IEEE International Conference on Communications (ICC 2008), pp. 3253-3257, May 2008.

[8] Z. Kong and Y.-K. Kwok, "VCG-Based Time-Slot Auctioning in IEEE 802.16 OFDM/TDMA Wireless Mesh Networks," Proceedings of the 5th International Conference on Wireless Communications, Networking and Mobile Computing(WiCOM2009), Sept. 2009.

[9] V. Krishna, Auction Theory, Academic Press, London, UK, 2002.

[10] L. E. Miller, Wireless Communications Technology Group, National Institute of Standards and Technology, Gathersburg, Maryland, "Formulas For Blocking Probability," Apr. 2002, http://w3.antd.nist.gov/wctg/netanal/BlckForm.pdf.

[11] D. Niyato and E. Hossain, "A Radio Resource Management Framework for IEEE 802.16-Based OFDM TDD Wireless Mesh Networks," Proceedings of the 2006 IEEE International Conference on Communications (ICC 2006), pp. 3911-3916, June 2006.

[12] D. Niyato and E. Hossain, "Integration of IEEE 802.11 WLANs with IEEE 802.16-Based Multihop Infrastructure Mesh/Relay Networks: A Game-Theoretic Approach to Radio Resource Management,"IEEE Network, vol. 21, no. 3, pp. 6-14, May-June 2007.

[13] M. J. Osborne, An Introduction to Game Theory, New York: Oxford University Press, 2004.

[14] J. Sun, E. Modiano, and L. Zheng, "Wireless Channel Allocation Using an Auction Algorithm," IEEE J. Select. Areas Communications, vol. 24, no. 5, pp. 1085-1096, May 2006. 